\title{
Easing hospitalist electronic health record burden through clinical workstation single sign-on
}

\author{
George A. Gellert*1, Crystal Delacerda ${ }^{2,3}$, Lajja Patel ${ }^{4,5}$, Gabriel Maciaz ${ }^{6}$ \\ ${ }^{1}$ Clinical Transformation and Health Informatics Advisor, San Antonio, TX, United States \\ ${ }^{2}$ CHRISTUS-Children's Hospital of San Antonio, TX, United States \\ ${ }^{3}$ Baylor College of Medicine, San Antonio, TX, United States \\ ${ }^{4}$ CHRISTUS-Santa Rosa Medical Center Hospital, TX, United States \\ ${ }^{5}$ Medcede Inc., San Antonio, TX, United States \\ ${ }^{6}$ Department of Health Informatics, CHRISTUS Health Santa Rosa, San Antonio, TX, United States
}

Received: May 13, 2020

DOI: $10.5430 /$ jha.v9n3p24
Accepted: June 15, 2020

Online Published: June 24, 2020

\begin{abstract}
Background: Computer workstation single sign-on (SSO) was implemented in 19 hospitals to reduce manual keyboard login and expedite access to the electronic health record (EHR) and clinical applications.

Objective: To quantify hospitalists time liberated from EHR keyboard to focus on patient care, and estimate financial value of this time for hospitalists.

Methods: Login duration prior to and after SSO implementation were compared in eight hospitals. Using national estimates of hospitalist hourly wage, dollar values of time liberated from keyboard were calculated, stratified by different levels of total EHR use.

Results: Following SSO implementation, first of shift login decreased 5.3 seconds (15.3\%), and reconnect duration decreased 20.4 seconds (69.9\%). The volume of hospitalist EHR use among all physician end users comprises $70 \%-90 \%$ of all electronic documentation and clinical orders issued, yielding an annual range of 10,302 hours (or 858.5 12-hour shifts) to 13,245 hours (or $1,103.812$-hour shifts) in hospitalist time liberated from keyboard for patient care, with recurrent annual value of $\$ 1,164,126$ to $\$ 1,496,685$.

Conclusions: Hospitalists gained meaningful amounts of time for patient care from SSO implementation. This time accrued to substantial financial value. SSO eases the EHR burden of hospitalists, and facilities using hospitalists extensively should consider SSO implementation.
\end{abstract}

Key Words: Computer workstation login, Single sign-on, Clinical workstation security, Hospitalist workflow, Electronic health record, Hospitalist EHR workflow, Physician EHR burn out

\section{INTRODUCTION: THE HOSPITALIST'S EHR BURDEN}

Hospitalists are central drivers and coordinators of multidisciplinary patient care delivery in the U.S., with $75 \%$ of U.S. hospitals now employing over 50,000 hospitalists. ${ }^{[1]}$
Hospitalists have also emerged as among the heaviest, most frequent users of the electronic health record (EHR) and certified or computer provider order entry (CPOE). ${ }^{[2]}$ Hospitalists may spend as much as $33 \%$ of their work day, or almost four hours, actively working in the EHR, ${ }^{[3]}$ and clinical docStates.

*Correspondence: George A. Gellert; Email: ggellert33@gmail.com; Address: San Antonio, 703 Sentry Hill, San Antonio, TX 78260, United 
umentation time has increased from $16 \%$ to $28 \%$ with EHR implementation. ${ }^{[4]}$ In many U.S. facilities, hospitalists are clinical team leaders from whom much clinical care delivery and treatments flow.

High physician dissatisfaction with EHR usability, interfaces, navigation, interruption of cognitive processes in care delivery and workflows is well documented. ${ }^{[2,5-10]}$ Concerns include the added time demands placed by EHRs on hospitalists already managing high patient volumes in busy shifts and ensuring patient safety. ${ }^{[3,5,9,10]}$ Time spent on the EHR is viewed as competing for time to deliver patient care and attend to patients' clinical needs. ${ }^{[2-5,11]}$ Health information technology related stress and burnout have also been reported. ${ }^{[6,7,12-15]}$ Using the EHR involves many repeated logins to ensure the security/confidentiality of patients' personal health information. Password protection of EHR access and clinical applications, requiring continual updating of complex passwords, adds to this burden, with the heaviest EHR users such as hospitalists impacted most negatively.

\section{REDUCING HOSPITALIST EHR LOGIN TIME WITH SINGLE SIGN-ON}

CHRISTUS Health is an independent delivery network comprised of 49 hospitals and over 300 other care facilities, with over 15,000 affiliated physicians. In most CHRISTUS hospitals, the hospitalist team leads patient care delivery, issues the greatest volume of care orders and completes the most electronic clinical documentation. ${ }^{[16]}$ Single sign-on (SSO) is a technology which enables a hospitalist or other clinician to login by the usual manual method on a keyboard at the start of shift, and then deploys a streamlined method using a proximity card/badge and a card reader at EHR workstations to expedite reconnects on all subsequent logins for the remainder of that shift. The technology reduces manual keyboard typing to login to just once per 12-hour shift, and accelerates subsequent access to the EHR. SSO also eliminates need to create and remember multiple complex passwords, and use of "cheat sheets" listing their passwords for different clinical software applications (a security risk to patients' personal health information).

Most hospitalists in the CHRISTUS system roam across their facility's units and service lines, and manage substantial patient volumes and throughput. Roaming hospitalists gain rapid access to information systems at the point of care with SSO. CHRISTUS Health implemented SSO technology in order to provide hospitalists and other clinicians with expedited access to the EHR and clinical applications, and to eliminate wasted time diverted from clinical care logging and managing passwords. The SSO proximity badge logs

Published by Sciedu Press hospitalists into and out of the EHR for 12-hour shifts as they roam throughout the hospital. When they tap the reader to reconnect to the EHR, SSO brings them back to precisely where they last left off in the EHR. When hospitalists move to another clinical workstation, the badge reader at each workstation enables them to access the current state of the last workstation they used.

Numerous clinical software applications can be accessed with SSO, and new applications can be profiled and deployed rapidly. Automated application password change processes ease the burden of password management. SSO automatically launches and opens applications. When the hospitalist leaves the workstation, the proximity badge automatically locks and secures it. The need to manually lock sessions is eliminated, as is the risk of losing work in the EHR when a hospitalist is distracted or diverted, and thus SSO also helps prevent disruptions to EHR workflow.

In implementing SSO, CHRISTUS Health migrated from workstation personal computers (PCs) to a thin client, a smaller, less costly device which enables connectivity to a remote server where processing occurs rather than within a local desktop PC at the clinical workstation. A virtual desktop replaced physical PCs so hospitalists could roam widely, and 45 clinical software applications were enabled for SSO. The shift to a thin client device in the workstations also saved resources because their replacement cost is \$200 versus $\$ 1,000$ for a PC.

CHRISTUS Health regarded SSO technology as a method to rapidly ease and expedite EHR and clinical application access for hospitalists and other high volume EHR users, and to improve EHR usability. The purpose of this analysis was to identify the SSO time savings benefit to hospitalists by quantifying the annual reduction of hospitalist keyboard typing conveyed by SSO, and to estimate the financial value of this time for the highest volume physician EHR end users. While the financial value of hospitalist time reduction in keyboard typing does not return to the system budget and enable physician workforce reductions, it does enable clinicians to spend more time focused on delivering patient care rather than typing on a keyboard (an activity with no clinical care value), and in this manner may expedite patient throughput and deliver a return on investment.

\section{METHODS}

\subsection{Study setting}

CHRISTUS Health is an independent delivery network, and SSO was used to expedite login among physicians, midlevel providers, nurses, respiratory therapists, dieticians and physical therapists who roam within 19 community hospitals 
which vary in licensed bed capacity from approximately 80 to 170 beds. The EHR in these facilities was MEDITECH Client Server Version 5.66, and the SSO product implemented was Imprivata OneSign Version 5.1.

\subsection{Study design}

Data on clinician time liberated from keyboard by SSO was collected and then the amount of time saved was converted into estimates of the dollar value of this time based on national estimates of hospitalist hourly wage. Hours and 12hour shift equivalents of time saved by hospitalists in reduced login and reconnect activity were converted into total dollar value produced when clinicians are liberated to care for patients, rather than typing on a keyboard to access the EHR and clinical applications. Weekly and annual time savings, and associated weekly and annual dollar values of time saved were calculated at the enterprise and individual facility levels.

The SSO software enabled precise quantification of the number of logins/reconnects by physicians, but at the time of this study not specific specialties such as hospitalists versus other specialties. The SSO product deployed, Imprivata OneSign Version 5.1, and Citrix virtualization software provide data on real time and retrospective SSO logins, including number of clinical users and frequency of access by application and per unit of time.

\subsection{Sampling techniques}

Mean login time duration before and following SSO implementation was measured over 128 sampled logins during two seven-day periods across eight hospitals selected randomly from 19 facilities. SSO access involves two logins: first login of the shift to the desktop and then EHR, and subsequent reconnect or re-logins to the EHR using the proximity card reader. Seven-day observation periods of login duration before and following SSO implementation involved sampling in April 2018 at the eight randomly selected facilities.

One hospital was sampled from each of the eight operational regions comprising the CHRISTUS enterprise. Facilities selected for sampling did not differ meaningfully from the rest of the 19 facilities for SSO performance, and login and reconnect times within any cluster of hospitals were historically very similar. Measurements of actual login and re-login/reconnect durations to workstations were completed before and following SSO implementation. A total of 128 login time durations were measured during day shifts, when EHR use and system demand are greatest. Workstations were sampled on different floors and service lines of each facility. There was no systematic variation or changes evident in SSO time to login performance between repeated samplings or in different facilities.

\subsection{Sample size}

There was an average of 184,606 logins to the enterprise EHR by all multidisciplinary clinicians across 19 facilities over two seven-day periods sampled pre- and post-SSO implementation in April 2018. Total potential SSO end users across the enterprise equaled 27,667, of whom 16,840 were enrolled for SSO use $(60.9 \%)$. Of these, 12,903 or $76.6 \%$ were clinicians actively using SSO in the 19 hospitals. Thirty percent of active clinicians or 3,871 were physicians.

\subsection{Data collection tools}

Citrix Studio was the virtualization analytics tool utilized, and manual reports were generated from the Imprivata OneSign appliance. Evaluation and timing of sample login times were completed manually on workstations at each hospital. Mobile devices were excluded due to large variation in their set up and deployment.

\subsection{Data analysis and management}

Mean first-of-shift login duration and mean reconnect login duration during the rest of shift were compared prior to and post-SSO implementation. Individual facility and enterprisewide clinician time liberated from keyboard were quantified and are reported in hours and the dollar value of hospitalist professional time, per week and per year. Mean login duration was multiplied by number of total first of shift and subsequent reconnects or re-logins. The total time required for hospitalists to login pre-and post-SSO implementation are reported at percent deciles of total EHR and clinical application use, as hospitalist CPOE order issuance and electronic documentation completion rates serve well as proxies for hospitalist login frequency.

The financial value or benefit resulting from decreased hospitalist login times (time liberated from keyboard for patient care) has been calculated using a hospitalist hourly wage estimate of $\$ 113$ per hour, derived from the mean of five national wage reporting sources. ${ }^{[17-21]}$ A seven on/seven off schedule of hospitalist shifts was used, resulting in a total of 2,184 hours worked per year. Notably, the dollar savings or value of the time liberated from keyboard for hospitalists by SSO are not resources reverting back to the hospital budget, nor do they enable less hospital spend on hospitalist headcount or salaries per se. However, hospitalist time liberated from keyboard is nonetheless valuable because this is added time that hospitalists can focus on patient care delivery instead of keyboard EHR login, and can thus indirectly facilitate increased patient throughput and, potentially, care quality due to a less distracted workflow. 


\section{Results}

During the seven-day observation periods in April of 2018, clinicians logged in to the EHR an average of 184,606 times as shown in Table 1. Prior to SSO implementation, the first of shift login to the Windows desktop required a mean duration of 30.1 seconds, plus 4.5 seconds to access the EHR, for a total of 34.6 seconds. The duration of reconnect/re-login by keyboard typing for the rest of the shift after first login was an average of 29.3 seconds. Once SSO was implemented, duration of first of shift individual login decreased $5.3 \mathrm{sec}$ onds, or by $15.3 \%$. Subsequent reconnect or re-login to the EHR was reduced by 20.4 seconds or $69.6 \%$ to an average of 8.9 seconds.

Table 1. Single sign-on reduction of login time among all clinicians in 19 hospitals

\begin{tabular}{|c|c|c|}
\hline Login Performance Parameter & $\begin{array}{l}\text { Enterprise-Wide } \\
\text { Frequency or Time }\end{array}$ & $\begin{array}{l}\text { Enterprise-Wide Keyboard Time } \\
\text { Reduction Enabled by SSO }\end{array}$ \\
\hline Total number of logins to the EHR over 7 days & 184,606 & -- \\
\hline Number of clinical users of single sign-on & 12,903 & -- \\
\hline \multicolumn{3}{|l|}{$\begin{array}{l}\text { Time duration of first of shift logins over } 7 \text { days for } 19 \\
\text { hospital facilities }\end{array}$} \\
\hline Pre-SSO & 235.2 hours (19.6 shifts) & 36.0 hours $\left(3.0\right.$ shifts $\left.^{*}\right)$ \\
\hline Post-SSO & 199.2 hours (16.6 shifts) & \\
\hline \multicolumn{3}{|l|}{$\begin{array}{l}\text { Time duration of workstation reconnects over } 7 \text { days for } \\
19 \text { hospital facilities }\end{array}$} \\
\hline Pre-SSO & $1,303.3$ hours (108.6 shifts) & 907.4 hours (75.6 shifts) \\
\hline Post-SSO & 395.9 hours (33.0 shifts) & \\
\hline Annual time savings enabled by SSO all clinicians & 19 hospitals & $49,056.8$ hours $(4,088.1$ shifts $)$ \\
\hline Annual time savings per facility all clinicians & 1 facility & 2,584.4 hours (215.4 shifts) \\
\hline Annual SSO time savings physicians only & 19 hospitals & 14,717 hours $(1,226.4$ shifts $)$ \\
\hline Annual SSO time savings physicians only per facility & 1 facility & 774 hours (64.5 shifts) \\
\hline
\end{tabular}

Note ${ }^{*}$ Shifts $=$ shift equivalents of 12 hours duration

Over the seven-day observation period, the sum total frequency of subsequent re-logins by clinicians was 160,134 over all 19 hospitals, or a total of 395.9 hours of EHR reconnect time (see Table 1). The reduction of total clinician reconnect/re-login time over a seven-day period was calculated as 1303.3 hours prior to SSO implementation minus 395.9 hours after implementation, demonstrating a net gain of 907.4 hours of multidisciplinary clinician time that was liberated from keyboard typing across the enterprise (which equals 75.6 shift equivalents of 12 hours per shift).

With first of shift login savings of 36 hours per week, the total weekly time savings across 19 facilities is 943.4 hours or 78.6 12-hour shifts, equaling 49.7 hours or 4.1 12-hour shift equivalents on average in each facility. Over the course of a year per facility, SSO produces 2584.4 hours (215.4 12-hour shifts) of total physician, nursing and ancillary clinician time that is freed from typing on the EHR keyboard.

For all 19 hospitals, 49,056.8 hours of clinician time (physicians, nurses and ancillary) are freed or from typing on the EHR keyboard, equal to 4,088.1 12-hour shift equivalents (see Table 1). Of this, SSO specifically liberated 14,717 hours of physician time from keyboard across 19 facilities per year, or 774 hours per facility per year (see Table 1). Because individual hospitals vary in the extent to which hospitalists are deployed and in their use of the EHR and clinical applications, we report the time liberated from keyboard stratified by increasing deciles of percentage use exclusively by hospitalists (see Table 2), for both the average individual facility and for all 19 hospitals implemented. At each tier of hospitalist use of the EHR, which serves as a proxy for login frequency, the annual financial value of time liberated from keyboard for an individual facility and for all 19 facilities are reported, using a mean hospitalist hourly wage of $\$ 113$.

For most of the 19 hospitals evaluated, and based on past usage, the volume of hospitalist EHR use and application logins as a component all physicians use would be $70 \%$ at the low end and as high as $90 \%$ across different facilities, yielding an annual range of hospitalist time liberated of 10,302 hours (or 858.5 12-hour shifts) to 13,245 hours (or 1,103.8 12-hour shifts). This time has an annual recurrent value of between $\$ 1,164,126$ and $\$ 1,496,685$, where hospitalists are liberated from keyboard to deliver patient care (see Table 2). 
Table 2. Financial value of hospitalist time freed from keyboard typing by SSO

\begin{tabular}{|c|c|c|c|c|}
\hline $\begin{array}{l}\text { Level of Hospitalist } \\
\text { CPOE Order } \\
\text { Issuance/EHR Use }\end{array}$ & $\begin{array}{c}\text { Hospitalist Time } \\
\text { Liberated Per Hospital } \\
\text { Per Year (Shifts) }^{\#}\end{array}$ & $\begin{array}{c}\text { Hospitalist Time Liberated } \\
\text { in } 19 \text { Hospitals Per Year } \\
\text { (Shifts) }^{\#}\end{array}$ & $\begin{array}{c}\text { Annual Value of Hospitalist } \\
\text { Time Liberated Per } \\
\text { Facility }^{\&}\end{array}$ & $\begin{array}{c}\text { Annual Value of Hospitalist } \\
\text { Time Liberated in } 19 \\
\text { Facilities }^{\&}\end{array}$ \\
\hline $30 \%$ & $\begin{array}{c}232 \text { hours } \\
(19.3 \text { shifts })\end{array}$ & $\begin{array}{c}4,415 \text { hours } \\
(367.9 \text { shifts })\end{array}$ & $\$ 26,257$ & $\$ 498,895$ \\
\hline $40 \%$ & $\begin{array}{l}309 \text { hours } \\
\text { (25.7 shifts) }\end{array}$ & $\begin{array}{l}5,887 \text { hours } \\
\text { (490.6 shifts) }\end{array}$ & $\$ 35,012$ & 665,231 \\
\hline $50 \%$ & $\begin{array}{c}387 \text { hours } \\
\text { (32.3 shifts) }\end{array}$ & $\begin{array}{l}\text { 7,358 hours } \\
(613.2 \text { shifts })\end{array}$ & $\$ 43,760$ & $\$ 831,454$ \\
\hline $60 \%$ & $\begin{array}{l}464 \text { hours } \\
\text { (38.7 shifts) }\end{array}$ & $\begin{array}{l}8,830 \text { hours } \\
(735.8 \text { shifts })\end{array}$ & $\$ 52,515$ & $\$ 997,790$ \\
\hline $70 \%$ & $\begin{array}{c}542 \text { hours } \\
\text { (45.2 shifts) }\end{array}$ & $\begin{array}{l}10,302 \text { hours } \\
(858.5 \text { shifts })\end{array}$ & $\$ 61,269$ & $\$ 1,164,126$ \\
\hline $80 \%$ & $\begin{array}{c}619 \text { hours } \\
\text { (51.6 shifts) }\end{array}$ & $\begin{array}{l}11,774 \text { hours } \\
\text { (981.2 shifts) }\end{array}$ & $\$ 70,024$ & $\$ 1,330,462$ \\
\hline $100 \%$ & $\begin{array}{l}774 \text { hours } \\
\text { (64.5 shifts) }\end{array}$ & $\begin{array}{c}14,717 \text { hours } \\
(1,226.4 \text { shifts })\end{array}$ & $\$ 87,527$ & $\$ 1,663,021$ \\
\hline
\end{tabular}

Note. ${ }^{*}$ Proxy for frequency of hospitalist EHR and clinical application logins as a percent of total physician logins; ${ }^{\#}$ Shifts $=$ shift equivalents of 12 hours duration; ${ }^{\&}$ Using $\$ 113$ per hour as the mean hourly wage of hospitalists, as derived from five national reporting sources.

\section{Discussion AND CONCLUSIONS}

Objectives for SSO implementation were met and exceeded, with a substantial liberation or reduction of clinician time logging in to the EHR and clinical applications. Hospitalists, as a group of heavy EHR users, may be the most significant beneficiaries of SSO. Even in facilities where only $60 \%$ of EHR use and logins are by hospitalists, the annual value of hospitalist time liberated from keyboard to deliver patient care approaches $\$ 1$ million per year on a recurrent basis, and climbs thereafter with each additional decile of hospitalist EHR use. With each $10 \%$ increase in hospitalist EHR use, SSO liberates another 1,472 hours (or 122.7 12-hour shifts) from keyboard, adding a financial value of $\$ 166,336$ across the 19 facilities implemented. We did not measure differences in day versus night shifts with respect to login frequency and thus SSO value, however the typical EHR usage pattern among CHRISTUS hospitalists involves significantly heavier use during the daytime shift, when most care is provided, most CPOE orders are issued, and most electronic documentation occurs.

Total one-time implementation cost for SSO was $\$ 1,230,000$, and the annual maintenance cost including clinician end user licenses is $\$ 296,000$, yielding a total first year SSO cost for these 19 hospitals of $\$ 1,526,000$. This analysis demonstrates that the dollar value of hospitalist time liberated from keyboard to deliver patient care - and to help drive patient throughput - almost equals the total one-time SSO implementation and recurrent annual maintenance cost. The savings on purchasing replacement PCs each year by migrating to a virtual desktop and a thin client device alone exceeds the annual SSO licensing fee. However, this calculus does not factor in the significant benefit of SSO to other physician specialists and mid-level providers, nurses and ancillary services, or in improving care team satisfaction and ability to focus on patient care. When these are considered, SSO value delivery exceeds its costs. While hospitalist satisfaction surveys were not collected, individual and chief hospitalists reported anecdotally that use of the EHR and clinical applications was easier and faster with SSO.

Overall, SSO provides an additional layer of encryption to the login and authentication process, and thus enhanced the level of personal health information security at the participating hospitals. The primary potential downsides of SSO implementation, depending on the implementation, include badge sharing among providers, or end users gaining access to a medical record that was previously open at a particular workstation where the last end user failed to log off. Both issues are readily addressed through end user training and establishment of policies to prohibit badge sharing, and requiring that end users tap out after completing their work or before even briefly leaving a workstation with a record open. In addition, ensuring an EHR auto log off is set after a certain time of inactivity reduces the existing low probability of accessing an open patient record.

Although hospitalists are not the final decision makers in whether a particular facility or system will invest in single sign-on, this analysis can raise awareness of the value of SSO among hospitalists so they can, through their usual participation in hospital governance, advocate for the value SSO would convey. Hospitalists sit on or lead important hospital committees where SSO implementation is considered, and have a critical role in maintaining/improving facility 
patient throughput and in decisions impacting EHR workflow. Furthermore, the literature on physician burn out in general, and on physician EHR burnout in particular, is expanding rapidly. ${ }^{[22]}$ Physician advocacy efforts in their own self-interest are hopefully growing as physicians increasingly insist that their voices on EHR and other burnout issues be heard. Often delivering a majority of patient care, hospitalists can advocate for EHR and other workflow improvements that impact professional well-being by requesting solutions like SSO. This analysis arms hospitalists (and hospital administrators) with data to advocate for improvements in EHR workflow, given how critical hospitalist performance is to meeting facility financial and clinical performance goals. Hospitalists and other clinical leaders at facilities where roaming across service lines is common should consider the value that SSO implementation may deliver to clinical workflow, clinician satisfaction, and in returning time to focus on the care of patients.

\section{ACKNOWLEDGeMENTS}

The authors are grateful to the clinicians of CHRISTUS Health, and to the members of the CHRISTUS Health Informatics and Information Management teams that supported the implementation of single sign-on.

\section{ETHICAL APPROVAL}

As no patients or identified health information were utilized in this study, no ethical approval or review was required.

\section{FUNDING SOURCE}

No funding supported this study.

\section{CONFLicts OF INTEREST Disclosure}

The authors declare they have no conflicts of interest.

\section{REFERENCES}

[1] Conrad K, Valovska T. The Current State of Hospital Medicine: Trends in Compensation, Practice Patterns, Advanced Practice Providers, Malpractice, and Career Satisfaction. In: Conrad K. (eds) Clinical Approaches to Hospital Medicine. Springer, Cham; 2018. PMid: 30140548. https://doi .org/10.1007/978-3-319-647 74-6_18

[2] Wachter RM. Annals for Hospitalists Inpatient Notes - Hospitals and Digital Medicine - Overcoming the Productivity Paradox. July 19, 2016 [Accessed at July 2, 2019]. PMid: 27429311. https: //doi.org/10.7326/M16-1367

[3] Kara A, Kok A, Bo N, et al. Hospitalists and the electronic medical record. Abstract published at Hospital Medicine. Orando, Fla. Abstract 290. April 8-11, 2018 [Accessed July 3, 2019]. Available from: https://www.shmabstracts.com/abstract/hospital ists-and-the-electronic-medical-record/

[4] Baumann LA, Baker J, Elshaug AG. The impact of electronic health record systems on clinical documentation times: A systematic review. Health Policy. 2018; 122: 827-36. PMid: 29895467. https://doi.org/10.1016/j.healthpol.2018.05.014

[5] Friedberg MF, Chen PG, Van Busum KR, et al. Factors affecting physician professional satisfaction and their implications for patient care, health systems, and health policy. Rand Health Q. 2014; 3(4): 1.

[6] Wachter R, Goldsmith J. To combat physician burnout and improve care, fix the electronic health record. Harvard Business Review. March 30, 2018.

[7] Medical Economics Staff. What's ruining medicine for physicians: Difficulty using EHRs. Medical Economics. December 11, 2018.

[8] Medical Economics, 2018 EHR Report Card. Medical Economics. October 22, 2018.

[9] Quinn R. Hospitalists' EMR frustrations continue: SHM report. The Hospitalist. May 9, 2017.

[10] Society of Hospital Medicine White Paper, Hospitalist Perspectives on Electronic Medical Records. HM17.

[11] Goroll AH. Emerging from EHR purgatory - moving from process to outcomes. New England Journal of Medicine. 2017. PMid: 28538132. https://doi.org/10.1056/NEJMp1700601
[12] Gardner RL, Cooper E, Haskell J, et al. Physician stress and burnout: The impact of health information technology. JAMIA. 2019; 26: 10614. PMid: 30517663. https://doi.org/10.1093/jamia/ocy1 45

[13] Flores L. America's physicians: Overworked and burning out. The Hospital Leader, Official Blog of the Society of Hospital Medicine. December 17, 2018 [Accessed at July 1, 2019].

[14] Sriram I, Harland R, Lowenstein SR. Perspectives in Hospital Medicine: I, EHR. Journal of Hospital Medicine. May 10, 2019.

[15] Wachter R. The Digital Doctor: Hope, Hype and Harm at the Dawn of Medicine's Computer Age. New York, NY: McGraw-Hill Education; 2015.

[16] CHRISTUS Health. CHRISTUS Health electronic health record clinical order issuance stratified by physician specialty, internal report. January 2012.

[17] Medscape, Medscape hospitalist compensation report. 2019 Available from: https://www.medscape.com/slideshow/201 9-compensation-hospitalist-6011429? src $=$ WNL_physrep _190608_lifestyle_hospcomp2019\&uac $=314034$ CV\&impID $=1$ 988103\&f af $=1 \# 12$

[18] Today's Hospitalist. 2018 Hospitalist compensation and career survey. Available from: https://www.todayshospitalist.com/ survey/18_salary_survey/index.php

[19] Salary.com. Physician-hospitalist salary in the USA. 2019. Available from: https://www.salary.com/research/salary/benchma rk/physician-hospitalist-hourly-wages

[20] PayScale. Average hospitalist (physician) salary. 2018. Available from: https://www.payscale.com/research/US/Job=Hospi talist_(Physician)/Salary

[21] ZipRecruiter. How much do hospitalist jobs pay per hour, 2019? Available from: https://www.ziprecruiter.com/Salaries/ How-Much-Does-a-Hospitalist-Make-an-Hour

[22] Jha AK, Iliff AR, Chaoui AA, et al. A crisis in health care: A call to action on physician burnout. Massachusetts Health \& Hospital Association. Massachusetts Medical Society, Harvard T.H. Chan School of Public Health and Harvard Global Health Institute. 2019. 